\title{
Research on the Formation and Cultivation of Psychological Resilience in Middle School Students
}

\author{
Zirou Liang \\ Guangdong Experimental High School, China
}

\begin{abstract}
Psychological resilience is of positive significance to the growth of adolescents. Teenagers with high psychological resilience can better overcome adversity and setbacks and pursue self-realization of life. Middle school students' psychological resilience is influenced by personal factors, family factors, school environment, and social factors. The level of psychological resilience can be improved by those activities such as strengthening the cultivation of individual positive personality traits, building a good social support system, developing resilience intervention training, implementing challenging and experiential teaching, strengthening psychological frustration education and social practice, and creating a positive campus cultural environment.
\end{abstract}

Keywords: middle school students; psychological resilience; training strategies.

\section{Introduction}

Psychological weaning refers to the transition period from childhood to maturity in the period from adolescence to early youth. The psychological development level of middle school students is in a semi-independent, semi-dependent, semi-mature and semi-immature "psychological weaning period". At this stage, while their physiology begins to develop and change, their personality also begins to form and develop, especially in mental characteristics such as intelligence, emotion, and will. On the one hand, it is because the mental development of middle school students is not yet fully mature and stable. Although their autonomy and independence consciousness is gradually increasing, they have the characteristics of naivety, impulsiveness, and unbalanced physical and mental development. On the other hand, it is because middle school students have changed from a relaxed and pleasant primary school to a tense and serious secondary school, facing a substantial increase in learning content and a change in learning methods. Many problems, such as change and brand-new interpersonal relationship management, which come from the pressure and frustration of life and learning, tend to make the middle school students have strong psychological conflicts and pressures. The ability to cope with such negative events as stress, adversity or trauma is "resilience" [1]. Psychological resilience is of positive significance to the growth of adolescents. Teenagers with high psychological resilience can better overcome adversities and setbacks and pursue self-realization of life.

\section{The Concept and Mechanism of Psychological Resilience}

As far as the definition of psychological resilience is concerned, the academic community has not yet reached a unified standard of recognition. Different scholars give different definitions from different angles. So far, the vast majority of studies on psychological resilience have identified two main operational defining elements: (1) individual encounter stress; (2) individual successful coping. [2] In 2005, American psychologists defined mental resilience as a good adaptation to life stresses, traumas, tragedies, threats or other major stresses, as a "resilience" to life stresses and setbacks.

Although many researchers have a different understanding of psychological resilience, most of them agree with the two main mechanisms of psychological resilience: internal personality and external environment. That is to say, in the process of formation and development of psychological resilience, various protective factors of "internal" and "external" play an important intermediary role. They reduce the possibility of negative consequences by interacting with various risk factors. [4] Broadly speaking, the so-called protective factors refer to those factors that can help adolescents improve their coping ability, help them overcome adversity, alleviate various negative effects and promote their positive development. In this sense, protective factors are buffer zones of pressure, which can reduce the degree of injury to individuals and make them able to withstand greater pressure 
by rebounding. Internal protective factors mainly refer to the positive psychological characteristics of individuals. For example, positive temperament, strong internal control, high level of self-esteem, high problem-solving skills, optimistic attitude towards the future and strong interpersonal skills, etc. [5]. External protective factors mainly include positive factors from family, school and community, such as a good parent-child relationship, harmonious peer relationship, positive school atmosphere and a sense of belonging and harmonious community environment, etc. Risk factors can also be divided into two categories: internal genetic factors and external environmental negative factors such as family, school and community. These factors include impulsive personality, low IQ and education level, poor parenting style, tense parental relationship, the conflict between parents, broken families, large population families, and poor community environment, etc.

\section{Factors Influencing Middle School Students' Psychological Resilience}

\subsection{Personal Factors}

The influence of personal factors mainly refers to the restrictions brought by congenital factors, which make people unable to achieve their goals smoothly. Psychological resilience is closely related to personal traits, and different personality traits have different abilities to cope with stress or difficulties. Generally speaking, sensitive and introverted individuals are prone to anxiety, pessimism, and disappointment when facing difficulties or adversities. They are depressed in the face of adversity and lack courage and perseverance to get out of the predicament and lose the motivation to move forward. When faced with setbacks, individuals with sound personality, positive mentality and optimism can actively adapt to and adjust their negative emotions, and make themselves stronger.

\subsection{The Influence of Family Factors}

The majority of contemporary middle school students are only children. Their family environment is generally superior, so they experience fewer hardships and setbacks, being lack of proper adaptability, and their psychological endurance is relatively weak. When facing complex interpersonal relationships and changes in the social environment, they are prone to negative emotions, which lead to stress reactions. Moreover, most parents expect their children to be excellent in all aspects of the school. They only pay attention to their children's academic achievements but neglect the cultivation of their children's ability to resist pressure. When children encounter adversity or setbacks, they take the initiative to take responsibility for their children, thus depriving them of the opportunity to grow up in various adversities. In addition to the current complex social situation, there are an increasing proportion of secondary school students who are staying behind, having divorced and single-parent families. Their families are not harmonious, their parent-child relationship is not good, they often lack parental care and emotional support, their personality is more sensitive and isolated, and they lack the sense of security and trust in their interaction with others, which negatively affects the development of psychological resilience.

\subsection{The Impact of School Environment}

If the family is the first environment for students' growth and development, then the school is the most important environment for students' growth and development. Since students spend most of their time here from the beginning of their enrollment, the quality of the school environment will directly affect the normal development of students' cognition and personality. A good school environment can not only stimulate students' initiative in learning but also effectively exercise students' strong will and cultivate a firm psychological quality. For a long time, our middle schools only pay attention to imparting students' knowledge, often neglecting the cultivation of students' positive personality quality, and lack the necessary intervention and training of students' psychological resilience. This often results in a considerable number of middle school students' poor psychological resilience, weak willpower, lack of indomitable spirit and adaptability to pressure in the face of difficulties or adversities. It leads to poor psychological resilience and adaptability. It is worth noting that the quality of teachers also plays a certain role in the impact of the school 
environment. Rutter, for example, found that children who grew up in unfavorable family environments could also have a strong level of psychological resilience if they met friendly and meticulous teachers at school. [6]

\subsection{The Influence of Social Factors}

Social factors play a subtle role in the development of middle school students' psychological resilience. With the gradual maturation and development of middle school students' minds, their psychological needs, such as security, love, belonging, respect, challenges, and talents, are increasing and eager to be met. However, some social conditions such as poverty, legal protection, and assistance, social acceptance of special adolescents and other influenced factors are difficult to ameliorate solely through individuals' or parents' efforts; rather, they often require the active involvement of states and relevant social organizations or groups. If the society has a relatively perfect juvenile protection system, providing specific services such as economic assistance fair access to education, and good social security defense for the adolescents in need, the development of their psychological resilience will promote to a large extent.

\section{Training Strategies of Psychological Resilience}

Although psychological resilience is determined to some extent by individual genetic or genetic factors, it is more related to acquired education and training. As a psychological trait that individuals use protective factors to cope with stressful situations and pursue personal self-development and selfrealization, there are many ways and means to excavate and train in practical operation.

\subsection{Strengthening the Cultivation of Individual Positive Personality Traits}

Relevant research shows that resilience, as a psychological trait, is closely related to positivity, optimism, self-confidence and other good personality traits, which directly affects the improvement of middle school students' psychological resilience level. Therefore, schools should pay special attention to consciously strengthening the cultivation and exercise of students' positive personality traits. On the one hand, it is important to cultivate the positive personality of a middle school student. Educators need to note that what degree of potentials that middle school students can stimulate when facing with various stressful events, and pay attention to cultivating middle school students' selfconfidence, optimism, positivity, courage and other strong personality traits of middle school students in learning and life. For example, by organizing students to forwardly participate in various meaningful activities, such as speech competitions, sports competitions, thematic discussions, and adversity imagination, we can guide middle school students to actively discover their own advantages from activities and receive respect from others and social recognition when giving play to their own advantages so as to achieve a sense of accomplishment. On the other hand, it is also important to educate students to improve their inner psychological quality and ability. It is important to let students learn to see all kinds of setbacks from life a positive attitude, and let them form good communication and problem-solving skills, as well as learn to establish an optimistic and confident attitude towards life. By fully tapping their potential and strength, they can play their subjective initiative to enhance their ability to resist pressure and shape positive personality traits, thereby enhancing psychological resilience and realizing the value of life. In this regard, the foundation of developing students' psychological resilience is the cultivation of their positive personality traits.

\subsection{Building a Good Social Support System}

As a backup system, social support can help individuals when they are under pressure or facing adversity, providing strength and courage for them to overcome difficulties and move forward. This kind of support can come from family, school, community, or other aspects of society. It has a significant protective effect of maintaining and developing positive attitudes under pressure or adversity in individuals. [7] Harmonious family atmosphere, good parent-child relationship, intimate peer relationship and positive guidance of teachers mentioned above all contribute to the formation 
of positive psychological characteristics of middle school students. For example, a good parent-child relationship and a warm family atmosphere can provide middle school students with a sense of security and trust. Relevant studies have shown that meeting the individual's psychological needs such as security, belonging, love and respect in the process of growth can help individuals establish a sense of security in the surrounding environment and an optimistic belief in future life, cultivate their self-confidence and strong personality traits, and enable them to adapt to setbacks and stressful environments effectively and efficiently. Therefore, parents should be good at providing encouragement and support to children in trouble, rather than just scolding and teaching. What's more, as a model and learning object of students, teacher's teaching can guide students to view all kinds of problems with a positive attitude in their learning and life, enhance their confidence and ability to deal with adversity correctly, and improve their adaptability to adverse situations. In addition, peer interaction is also one of the most important resources to promote the development of psychological resilience. Harmonious peer relationship is crucial to an individual's support. In the face of difficulties or adversities, talking with peers can not only quickly alleviate negative emotions but also reduce the negative impacts of negative emotions. At the same time, we can receive sincere and effective support and help from peers to, again, enhance the courage and self-confidence to face adversities; the more frustrated the condition is, the braver they become.

\subsection{Developing Resilience Intervention Training}

Generally speaking, psychological resilience intervention training is the most effective way to cultivate middle school students' psychological resilience. The so-called psychological resilience intervention training refers to the change of beliefs and ways of thinking to guide students to form an optimistic style of behaviors, improve the ability of individuals to think from multiple perspectives, thereby further improving students' social ability and their ability to cope with stress crisis. There are many models of intervention training, among which the IRO resilience training in Canada, "I am, I have, I can" operational training and six strategies training plan, have been proved to be an effective way of improving individual psychological resilience.

RI (Reaching In) in RIRO means "achieving", which aims to help individuals think more flexibly and correctly. RO (Reaching Out) means "going out", which aims to help improve their ability to interact with others and seize opportunities. RIRO resilience training aims at improving trainees' processing skills of resilience recovery after frustration. The training is usually divided into three parts. The first part is education. In this process, instructors disseminate the concepts of psychological resilience and resilience intervention training to let participants understand various related factors of psychological resilience. The second part is the operation. Trainers use the learning of various guidance programs to help participants enhance their resilience through this procedure. The third part is the summary. That is to say, based on previous practices of learning and training, instructors summarize the connotation of RIRO so that participants can consolidate their understanding. [8] Generally speaking, through training, trainees' beliefs and ways of thinking are guided to change, so that they can form an optimistic style of work, improve their ability to diverge their thinking from multiple perspectives, and ultimately achieve the goal of improving trainees' social ability and ability to cope with stress crisis.

"I am, I have, I can" is a well-known operational training. "I am" refers to the objective and positive understanding of oneself and the discovery of the internal strength of an individual. "I have" refers to the proper recognition of social support and resources that I have, reflecting on its shortcomings, promoting its strengths, and avoiding its weaknesses. "I can" refers to helping individuals to discover and develop interpersonal communication and problem-solving skills so that they can enhance selfconfidence and tap potential. [9] The combination of these three factors, on the one hand, promotes students to form correct and good self-perception; on the other hand, it enables students to recognize their weaknesses, set feasible goals, inspire their potentials, and actively respond to challenges.

There are six main implementation principles for psychological resilience intervention training, called "six strategies". One of them is to provide students with opportunities to participate actively in meaningful activities; another one is to establish and maintain high expectations of students; the third 
one is to create a good school atmosphere of mutual love and support; the fourth one is to formulate clear and consistent codes of conduct for students; the fifth one is to strengthen the pro-social tendency of each student; the sixth is to teach students various life and social skills.[10] In addition, to encourage middle school students to participate in the six strategies of mental resilience training organized by the school and to improve their mental resilience through their own efforts to obtain successful experiences, the school can organize some outdoor psychological quality development activities throughout the school and encourage students to participate actively, to experience the challenges of life through real experience, and to cultivate a positive and enterprising attitude towards life.

\subsection{Implementing Challenging and Experiential Teaching}

With the development of era and society, some traditional teaching methods cannot meet the growing needs of students, and some new teaching methods emerge due to social demand. Challenge teaching is a new teaching method that pursues development and creativity. It means that teachers constantly raise new challenges according to the latest development of a certain subject, to arouse students 'curiosity and enthusiasm; thus, they can stimulate students' potentials. Generally speaking, the challenges created by teachers should be completed within the given time and have certain difficulties to stimulate students' inner potentials. When operating challenge teaching, instructors should encourage students to explore different feasible solutions when dealing with problems, which practices their divergence thinking; second, they should cultivate students' ability to think and solve problems independently and firmly; third, teachers should be supporting students regardless of whether they can finally challenge successfully or not, avoiding damaging their enthusiasm. Therefore, the use of challenging teaching can not only motivate students to learn and improve their learning efficiency but also help to cultivate students' courage and confidence when experiencing new things and facing setbacks.

Experiential teaching is characterized by creating situations to arouse students' emotional experiences. Specific operation refers to creating a certain scene according to the teaching content and students' cognitive characteristics. Common forms of experiential teaching are role-playing, situational experience, transposition experience, and simulation of the scene, game, case analysis. Since experiential teaching can imitate the real scene in real life and enable students to obtain solutions to practical problems through their participation, it is beneficial for students not only to apply knowledge to practice, to develop their potentials through positive thinking and experiences, to realize self-education and self-growth, but also to effectively mobilize students 'enthusiasm for participation. Experiential teaching not only encourages students to practice and experience actively but also develop practical skills to cope with difficulties while making psychological preparations.

\subsection{Strengthening Frustration Education and Social Practice of Middle School Students}

Through carrying out special frustration education courses for middle school students, we can guide middle school students to face problems with positive attitude and optimism, explore creative solutions actively, experience happiness that life brought us, enhance self-confidence and perseverance, ultimately strengthen their resilience to difficulties or adversities. In addition, we can improve their ability to cope with various stresses, adversities, traumas and other negative events encountered in their studies and life, and enhance their psychological resilience. [11] At the same time, schools should organize and encourage middle school students to actively participate in various social practices, because practical activities can provide them opportunities to not only experience frustration but also effectively enhance psychological resilience. By volunteering in welfare homes and nursing homes, participating in street sanitation and other community organization activities, middle school students can experience hardships, strengthens their will, and eventually, improve their psychological resilience. 


\subsection{Creating a Positive and Upward Campus Cultural Environment}

School is one of the main learning and activity places for middle school students. It is an important field to promote the healthy development of middle school students' psychological resilience. It plays a key role in the learning and psychological development of middle school students. A positive and upward campus cultural environment can improve students' self-confidence and self-identity, provide more opportunities for students to exercise and compete, and create a platform to show their interests and hobbies.

Educators can develop various propaganda and education in a targeted and planned way by formulating various relevant educational management measures, emphasizing the ideal norms of conduct. For example, by inviting experts from inside and outside the school to hold special lectures and poster exhibitions, we can publicize the model figures and deeds with tough personality quality to create a positive campus atmosphere with high psychological resilience, so that middle school students can be inspired and encouraged to establish positive beliefs; we can also organize large-scale group activities with the theme of interpersonal communication and interpersonal trust to enhance students' sense of belonging and cooperation ability, promote the establishment of good interpersonal relationships, and let college students improve their psychological resilience through various aspects of development.

\section{References}

[1]. Zhang HO. Relationship between Self-Esteem, Coping Style and Psychological Resilience of Secondary Vocational School Students [J]. Special Education in China, 2012 (9): 77-81.

[2]. Zhou HN. Psychological Elasticity Research from the Perspective of Positive Psychology [J]. Journal of Ningbo Institute of Education, 2010 (5): 92-95.

[3]. Tousman, S., Zeitz H, Taylor LD. Development, Implementation, and Evaluation of a New Adult Asthma Self-Management Program[J]. Community Health Nurse, 2007, 24 (4): 237-251.

[4]. Ma W N, Sang B, Hong M M. A Review of Psychological Elasticity and Its Mechanism of Action [J]. Journal of East China Normal University (Educational Science Edition), 2008, 26 (1): 89-96.

[5]. Ruan W. The Positive Effect of Psychological Resilience in Middle School Students' Learning Burnout [J]. China Science and Education Innovation Guide, 2012 (15): 223-223.

[6]. Rutter M. Psychosocial Resilience and Protective Mechanisms[J]. American Journal of Orthopsychiatry, 1987, 57 (3): 316-331.

[7]. Yu X N, Zhang J X. Resilience - The Psychological Mechanisms of Recovery and Growth under Stress [J]. Progress in Psychological Science, 2005, 13 (5): 658-665.

[8]. Li X, Miao Y J. Training Practice of Psychological Resilience: Guidelines for Resilience of IRO in Canada [J]. Journal of Gannan Medical College, 2011, 35 (5): 792-794.

[9]. Zhang H L. The Relationship between Resilience and Mental Health of College Students [D]. Xi'an: Xi'an Petroleum University, 2012.

[10]. Wu Qiang, Ma Z Z. Positive Psychology and Its Educational Enlightenment [J]. Shanghai Educational Research, 2008 (6): 30-32.

[11]. Ge G Y. The Relationship between Middle School Students' Resilience and Academic Level [J]. Journal of Gannan Normal University, 2010 (1): 114-117. 